\title{
An Evaluation Model of Digital Educational Resources
}

\author{
http://dx.doi.org/10.3991/ijet.v8i2.2501 \\ El Mhouti Abderrahim, Erradi Mohamed, Nasseh Azeddine \\ Abdelmalek Essaadi University, Tetouan, Morocco
}

\begin{abstract}
Today, the use of digital educational resources in teaching and learning is considerably expanding. Such expansion calls educators and computer scientists to reflect more on the design of such products. However, this reflection exposes a number of criteria and recommendations that can guide and direct any teaching tool design be it campusbased or online (e-learning).

Our work is at the heart of this issue. We suggest, through this article, examining academic, pedagogical, didactic and technical criteria to conduct this study which aims to evaluate the quality of digital educational resources.

Our approach consists in addressing the specific and relevant factors of each evaluation criterion. We will then explain the detailed structure of the evaluation instrument used : "evaluation grid". Finally, we show the evaluation outcomes based on the conceived grid and then we establish an analytical evaluation of the state of the art of digital educational resources.
\end{abstract}

Index Terms-Criteria, Digital educational resources, evaluation grid, quality.

\section{INTRODUCTION}

Thanks to the possibilities offered by Information and Communication Technology (ICT) in education today, many multimedia and interactive educational products are elaborated within the framework of a campus-based or online education (e-learning).

In fact, the use of teaching devices and mediated communication is becoming a necessity. Such recourse involves double constraints. On the one hand, it involves mediating educational content and learning processes. In this case, the emphasis is on the importance of technological devices for mediation as well as different forms of mediation of their own. On the other hand, the use of technology involves the creation of specific forms of communication [1].

However, from a pedagogical and didactic perspective, the question now facing pedagogues and computer scientists is not whether to integrate digital pedagogy or not (its usefulness is quite evident and needs no reflection), but it is rather about the educational mission of digital resources [2]: Do these products really carry out their due tasks? (teaching and developing skills), or are they only electronic versions of traditional courses that will not bring anything special to the learner, who is sometimes disturbed by inadequate use of technology (choice of colors, number of links, the complexity of the interface)? What are the techno-pedagogical criteria to be taken into consideration for the development of digital learning resources in education?
Literature in educational multimedia offers many criteria and recommendations [3] that can guide and direct any digital design of educational resources in campus-based or online education. We point out, in a non-exhaustive way, the work of Crozat S., Trigano P. and Hû O. [4] ; Scapin D., and Bastien CH. [5]. However, these criteria are not always easy to implement or turn out to be very difficult to adapt in the case of customized products or small teams, which is true in most cases.

We propose in this work to contribute in this area by making an evaluation, followed by an analysis, of the inventory of digital learning resources used in teaching and learning, face or online. On the one hand, the study consists to develop an evaluation grid taking into account the academic, pedagogical, didactic and technical constraints. On the other hand, the study aims to evaluate digital educational resources by confronting them with different criteria and questions of the grid in order to identify possible results and analysis.

The purpose of our work is to come up with results that will help find out to what extent the conception of digital educational resources take into account the academic, pedagogical, didactic and technical aspects.

In other words, the study is intended to assist those in the field of education to evaluate the (pedagogical, didactic and technical) quality of digital, pedagogical and educational resources they use or intend to use.

\section{Methodology}

\section{A. Methodology}

To carry out our study, the initial step is to create a simple tool for the evaluation of digital educational resources. For this, we have adopted a systematic approach which consists in identifying the different aspects of this resources and describe each separately in its own context: academic, pedagogical, didactic and technical aspect. From the performed description and an exploration of conducted research in this field, we collect all the data in an "evaluation grid" to be rebuilt in a new context.

\section{B. Conception of the evaluation grid}

The adopted evaluation grid of a digital and educational resources has been designed to be easily used. It was developed using specific vocabulary to avoid multiple interpretations.

To identify the main criteria to be evaluated, the proposed approach consists in consulting a number of digital educational resources and visiting educational websites all dealing with the same subject, and then identifying elements which enable to compare and evaluate them [6]. 
In addition to the identification part and overview, used to identify and to present each evaluated product, the evaluation grid is built around four main relevant sections for the evaluation of both the content and the form of digital learning resources. These sections are "academic quality", "pedagogical quality ", "didactic quality" and "technical quality". Each section is associated with a set of additional criteria, and each criterion is then associated with one or more questions [7] to verify the suitability of the product examined with each reference criterion.

The evaluation grid must indicate if each product complies with the different criteria. For each question, referring to a criterion, we assign a score ranging from 0 to 5 on Likert scale ${ }^{1}$. The testing of different products using the grid provides quantitative results about its quality.

The grid we propose can serve as a reference for the elaboration of activities to develop digital educational resources . It is therefore a tool for pedagogy agents, who are concerned to design, but also to observe and analyze their teaching practices, in terms of the construction of digital educational resources. From this perspective that the grid was originally developed and tested in collaboration with professionals.

In the next part, we describe more precisely the different sections and criteria on which our evaluation grid is based.

\section{Description of the different sections}

\section{1) Product Identification}

This section identifies and presents a digital educational resource. In this part, we indicate the name or title of the product and identify the name of the authors or those responsible for the production in addition to the target audience.

\section{2) Academic quality aspect}

The objective of this section is to evaluate the quality of information presented in the digital learning resource. Indeed, the quality of information is an essential component of the experience the learners will be living by checking the content. There are two essential criteria to define the concept of quality applied to information:

- Information reliability :

Information reliability lies in credibility and accuracy. To evaluate this criterion, we start questioning whether the information is reliable, accurate and error-free. Is this accuracy sustainable over time? Is information security guaranteed? Is there any correspondence between the perceived reliability and the actual reliability of information?

- Information relevance :

This criterion is related to the effectiveness of information. We wonder if the information transmitted will trigger desirable behaviors for the learner. Is the information workable and usable?

These two elements (reliability and relevance) of academic quality are highly interdependent: the mechanisms implemented to ensure information reliability will obvi-

\footnotetext{
${ }^{1}$ Likert scale is a rating scale where the interrogated person expresses his/her degree of agreement or disagreement regarding a statement. The scale contains five or seven answer choices which enable to formulate the degree of agreement.
}

ously affect its relevance if the perceived reliability is good.

\section{3) Pedagogical quality aspect}

The evaluation of pedagogical quality is of paramount importance. To enhance learning and enable the learner to construct his/her knowledge, a digital learning resource must refer to a differentiated pedagogy, active and learnercentered which promotes the development of skills.

This section examines the various facets of the educational dimension brought by the digital learning resource. The main criteria that will face each product during the evaluation are:

- Pedagogical formulation :

Pedagogical formulation represents a concern of comprehension by learners who use digital educational resources for learning. This formulation is characterized by the quality of simplification, the presence of summaries or abstracts as well as the use of diagrams, figures and illustrations.

- Pedagogical construction :

Pedagogical construction evaluates whether the structure of the digital learning resource promotes its use in a pedagogical context [8] through the presence of appropriate interactivity, logic of organization, ease of orientation (eg summary, site plan), ease of browsing (back-forward, back to home page, scroll box) and readability of pages (internal summary, back buttons).

\section{- Pedagogical Strategies :}

This criterion evaluate the strategies of teaching and learning adopted. Developing an appropriate instructional strategy lies in designing and organizing learning activities based on techniques, methods, approaches and diverse educational models to handle different learning styles.

Teaching and learning strategies should be based on active teaching approaches (constructivism, socioconstructivism) to build meaningful and motivating situations for learners and engage them actively in learning.

- Assessment methods :

The assessment methods are tools implemented for evaluation, teaching, monitoring and learners' support, such as exercises and tests. This criterion aims to evaluate the assessment practices used. It also helps to ensure whether the assessment is promoted or opposes the emergence of learning.

\section{4) Didactic quality aspect}

Didactics focuses on the central role of learning activities, disciplinary content and epistemology (the nature of knowledge to be taught).

This section examines the didactic quality of pedagogical digital. We can define two key criteria to evaluate the quality of educational resources:

- Veracity of learning activities :

To enable the learner to manipulate the presented content, the activities proposed in the product must be appropriate. These activities must refer to real problems that could possibly face the learner outside the classroom.

- Content of the educational resource :

To achieve the learning objectives, the content of the product must be in adequacy with the objectives and target 
audience and knowledge conveyed must undergo changes without minimizing, deviating or affecting the concept.

\section{5) Technical quality aspect}

The technical quality of a digital learning resource is paramount. In fact, it is not acceptable that the learner will not be able to achieve an educational activity because of usage problems.

This section measures the resource elaboration from the perspective [9] :

- Design :

The content and organization of the visual product should promote appropriate use of colors, interactivity, graphic quality and pleasing aesthetic for the selected images and illustrations.

- Browsing :

The product design must facilitate browsing. While manipulating the resource, the learner should be able to find a plan, an index or a detailed table of contents. The suggested choices should be clear and the groupings within the menus should be consistent.

- Technological ingenuity :

Multimedia techniques aim to combine and exploit the capacities of new technologies in education to enhance knowledge transfer and assimilation of knowledge by learners.

During product development, the designer should use multimedia techniques in favor of information and education such as animations, flashing text, animated images and multiple windows.

Table 1 shows the designed evaluation grid.

\section{SAMPLE SELECTION}

\section{A. Sample}

After making the evaluation grid, we proceed with the evaluation of digital educational resources. In fact, testing such product involves making each face different criteria and questions of the grid.

The evaluation is performed on digital educational resources used within the Moroccan educational system context. This resources are produced by Moroccan companies $\left(\right.$ Eclisse $\left.^{2}\right)$, French (eduMedia ${ }^{3}$ ), etc..

In this context, and given the diversity and the large number of existing products, the study focused on a sample of 30 products representing all producers (national and international). This digital educational resources are used as part of secondary education.

\section{B. Work methods}

Evaluation is performed in the second task through matching selected products against the grid and giving scores. Pedagogical research and our teaching experience as a teacher for several years have enabled us to make this evaluation. In this regard, each product is displayed with a "data show". We put ourselves in the place of the end user of the product. We deal with each part one by one, and we, then, proceed to answer the 20 questions in the evaluation grid.

For each question in the grid, we have assigned a rating scale ranging from 0 to 5 (Likert scale) and to which, after having had an overview view of the whole product, we assign a score that we consider suitable for the selected question. The average or final grade is calculated according to the sum of scores for each product.

\section{RESULTS AND DISCUSSION}

In this section, the data will be presented and analyzed are related to the results of the two conducted evaluations. On the one hand, an overall evaluation of the quality of pedagogical digital resources is performed according to the rating scale. This first type of evaluation will allow us to have a general idea about the quality of the product evaluated. On the other hand, we have conducted an evaluation of the quality of each section (academic, pedagogical, didactic and technical).

\section{A. Global results of quality evaluation}

In this evaluation process, we adopt a rating method following Likert scale. The rating intervals show, for each average found, the quality level of the evaluated product:

- 81 to 100: The product is an excellent educational resource. It offers different functionalities and meets the required quality criteria;

- 61 to 80: the product includes some interesting elements despite some weaknesses;

- 41 to 60: the product category is average. It does not allow a sufficiently significant educational use;

- 0 to 40: the product is below the average. It does not meet several required (educational, scientific, technical) quality criteria.

The table II concerns the evaluation grid of the multimedia product "chute libre" of the chosen sample.

\footnotetext{
2 http://www.eclissecom.com

${ }^{3}$ http://www.edumedia-sciences.com/
} 
PAPER

An Evaluation Model of Digital Educational Resources

TABLE I.

QUALITY EVALUATION GRID OF DIGITAL EDUCATIONAL RESOURCES

\begin{tabular}{|c|c|}
\hline \multicolumn{2}{|r|}{ Evaluation grid of the quality of a digital learning resource } \\
\hline \multicolumn{2}{|r|}{ Identification and presentation of the product: } \\
\hline & -Target audience: \\
\hline \multicolumn{2}{|r|}{ Academic quality } \\
\hline Information reliability & 1. Is the information presented reliable? \\
\hline Information relevance & 2. Is the information presented relevant? \\
\hline \multicolumn{2}{|r|}{ Pedagogical quality } \\
\hline \multirow{2}{*}{ Pedagogical formulation } & 3. Is the quality of resource simplification good? \\
\hline & 4. Does the educational resource present overviews and summaries? \\
\hline \multirow{2}{*}{$\begin{array}{l}\text { Pedagogical construc- } \\
\text { tion }\end{array}$} & 5. The resource is clearly structured it (a plan or a summary, for example)? \\
\hline & 6. Structuring the resource she promotes its use in an pedagogical context? \\
\hline \multirow{8}{*}{ Pedagogical strategies } & 7. Are the objectives to be achieved stated? \\
\hline & 8. Does the product include stimuli likely to promote learning? \\
\hline & 9. Are knowledge and existing learners' representations taken into account? \\
\hline & 10. Is the active mental engagement of the learner favored? \\
\hline & 11. Is learning based on learner-centeredness? \\
\hline & 12. Are there any problem-solving tasks fostering a constructive learning? \\
\hline & 13. Does the tool present activities creating interactions between learners? \\
\hline & 14. Does learning allow anticipation of development by taking into account the $\mathrm{ZPD}^{4}$ ? \\
\hline Assessment method & 15. Does the tool provide an assessment procedure? \\
\hline \multicolumn{2}{|r|}{ Didactic quality } \\
\hline Learning activities & 16. Do activities refer to real problems which the learner will possibly facing outside the classroom? \\
\hline Learning content & 17. Is there a match between the audience, content and objectives? \\
\hline \multicolumn{2}{|r|}{ Technical quality } \\
\hline Design & 18. Is browsing between different elements of the product easy? \\
\hline Browsing & 19. Are multimedia techniques in favor of information and pedagogy? \\
\hline Technological ingenuity & 20.Do multimedia techniques promote information and pedagogy? \\
\hline
\end{tabular}
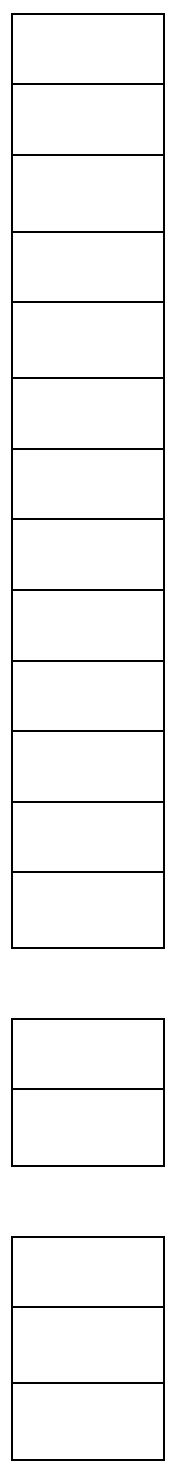

${ }^{4}$ Zone of Proximal Development "is the distance between the actual developmental level as determined by independent problem solving and the level of potential development as determined through problem solving, under adult guidance ". Vygotsky, L., 1978. 
PAPER

An Evaluation Model of Digital Educational Resources

TABLE II.

EVALUATION GRID FOR THE MULTIMEDIA TOOL « CHUTE LIBRE »

\begin{tabular}{|c|c|}
\hline \multicolumn{2}{|r|}{ Evaluation grid of the quality of a digital learning resource } \\
\hline \multicolumn{2}{|r|}{ Identification and presentation of the product: } \\
\hline \multicolumn{2}{|r|}{-Target Audience: Elèves de secondaire } \\
\hline \multicolumn{2}{|r|}{ Academic quality } \\
\hline Information reliability & 1. Is the information presented reliable? \\
\hline Information relevance & 2. Is the information presented relevant? \\
\hline \multicolumn{2}{|r|}{ Pedagogical quality } \\
\hline \multirow{2}{*}{ Pedagogical formulation } & 3. Is the quality of resource simplification good? \\
\hline & 4. Does the educational resource present overviews and summaries? \\
\hline \multirow{2}{*}{$\begin{array}{l}\text { Pedagogical construc- } \\
\text { tion }\end{array}$} & 5. The resource is clearly structured it (a plan or a summary, for example)? \\
\hline & 6. Structuring the resource she promotes its use in an pedagogical context? \\
\hline \multirow{8}{*}{ Pedagogical strategies } & 7. Are the objectives to be achieved stated? \\
\hline & 8. Does the product include stimuli likely to promote learning? \\
\hline & 9. Are knowledge and existing learners' representations taken into account? \\
\hline & 10. Is the active mental engagement of the learner favored? \\
\hline & 11. Is learning based on learner-centeredness? \\
\hline & 12. Are there any problem-solving tasks fostering a constructive learning ? \\
\hline & 13. Does the tool present activities creating interactions between learners? \\
\hline & 14. Does learning allow anticipation of development by taking into account the ZPD ? \\
\hline Assessment method & 15. Does the tool provide an assessment procedure? \\
\hline \multicolumn{2}{|r|}{ Didactic quality } \\
\hline Learning activities & 16. Do activities refer to real problems which the learner will possibly facing outside the classroom? \\
\hline Learning content & 17. Is there a match between the audience, content and objectives? \\
\hline \multicolumn{2}{|r|}{ Technical quality } \\
\hline Design & 18. Is browsing between different elements of the product easy? \\
\hline Browsing & 19. Are multimedia techniques in favor of information and pedagogy? \\
\hline Technological ingenuity & 20.Do multimedia techniques promote information and pedagogy? \\
\hline
\end{tabular}

\begin{tabular}{|c|}
\hline score $/ 5$ \\
\hline 5 \\
\hline 5 \\
\hline
\end{tabular}

\begin{tabular}{|c|}
\hline 3 \\
\hline 3 \\
\hline 4 \\
\hline 4 \\
\hline 3 \\
\hline 3 \\
\hline 3 \\
\hline 2 \\
\hline 3 \\
\hline 2 \\
\hline 2 \\
\hline 1 \\
\hline 3 \\
\hline
\end{tabular}

\begin{tabular}{|l|}
\hline 3 \\
\hline 3 \\
\hline
\end{tabular}

\begin{tabular}{|c|}
\hline 4 \\
\hline 5 \\
\hline 5 \\
\hline
\end{tabular}

- Name of the product evaluated: "chute libre";

- Subject: Physics Chemistry;

- Grade: Secondary ;

- Score of the final evaluation: 66/100.

\footnotetext{
${ }^{5}$ Ministry of National Education
} 
The table III presents the obtained results based on the adopted rating scale:

The above results provide us with information that may provide an idea of the quality of digital educational resources. The first information that can be drawn from these results is the existence of resources belonging to different intervals of the rating scale which means that quality levels vary from one product to another.

Firstly, despite some weaknesses (inadequacy with certain criteria), the majority of evaluated products $(36.67 \%)$ have interesting quality elements.

Second, a significant portion $(30 \%)$ of these products meets the needs of education and therefore has a satisfactory quality.

Other products $(23.33 \%)$ do not allow for educational use and are enough important and belong to middle category.

Finally, only $10 \%$ of the evaluated products do not meet several required quality criteria (educational, scientific, technical). These products are therefore below the average.

In fact, if one excludes the products (30\%) whose quality is satisfactory, the question that arises concerns the elements or rather the aspects (educational, academic, educational or technical) that led to quality with some medium or low weaknesses for the rest of the products $(70 \%)$. The answer to this question can be found in the results of the second evaluation.

\section{B. Evaluation results for each section}

We have chosen to focus on this second type of evaluation because we wanted to orient our analysis toward the impact that each section (academic, pedagogical, didactic and technical) could have on the quality of evaluated products. In this regard, to begin this evaluation, we proceeded as follows:

$>$ For each evaluated product, we calculate, for each section of the evaluation grid, the sum of the scores:

- section "academic quality": sum of scores/10;

- section "educational quality": sum of scores / 65;

- section "quality teaching": sum of scores / 10;

- section "technical quality": money notes / 15.

For the evaluated sample, we calculate, for each section, the average score and its representation in percentage.

The Table IV shows the obtained results for the evaluation of each section :

In this evaluation process, the results obtained separately for each of the four sections indicate that the digital educational resources evaluated have a good academic $(86.30 \%)$ and technical $(92.07 \%)$ quality . In contrast, we find that these resources do not meet the pedagogical $(45.49 \%)$ and didactic (41.90\%) needs and expectations.

These results allow us to answer the question we have obviously set which concerns the elements that led to a medium or low quality for a large number of evaluated products (results of the first evaluation: overall quality) . One of the explanations may be found in the weak pedagogical and didactic intervention.

The main conclusion we can draw from these two evaluations (global evaluation and evaluation according to each section) is that, despite their quality at the academic
TABLE III.

GLOBAL RESULTS ACCORDING TO THE ADOPTED RATING SCALE

\begin{tabular}{|c|c|c|}
\hline $\begin{array}{c}\text { Rating } \\
\text { scale }\end{array}$ & $\begin{array}{c}\text { Number of related } \\
\text { products }\end{array}$ & Representation in \% \\
\hline 81 to 100 & 9 & $30 \%$ \\
\hline 61 to 80 & 11 & $36.67 \%$ \\
\hline 41 to 60 & 7 & $23.33 \%$ \\
\hline 0 to 40 & 3 & $10 \%$ \\
\hline
\end{tabular}

Fig. 1 shows the representative histogram of the evaluation results according to the rating scale

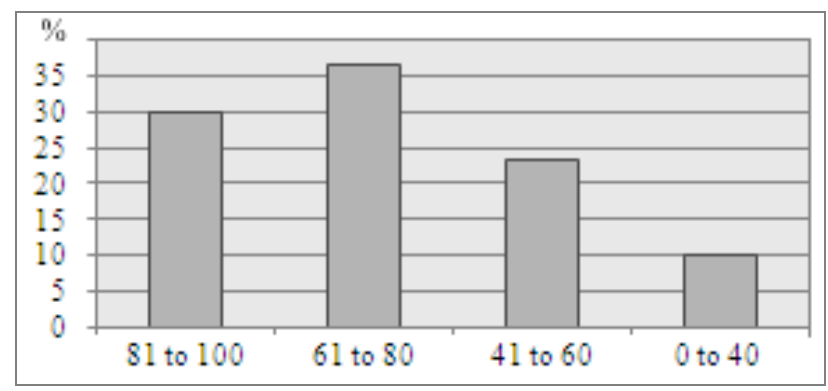

Figure 1. Representative histogram of global evaluation by the rating scale

TABLE IV.

RESULTS FOR THE EVALUATION OF EACH SECTION

\begin{tabular}{|l|c|c|}
\hline \multicolumn{1}{|c|}{ Sections } & Average score & Representation in \% \\
\hline Academic quality & 8.63 & $86.30 \%$ \\
\hline Pedagogical quality & 29.57 & $45.49 \%$ \\
\hline didactic quality & 4.19 & $41.90 \%$ \\
\hline Technical quality & 13.81 & $92.07 \%$ \\
\hline
\end{tabular}

Fig. 2 shows the representative histogram of the evaluation according to the sections:

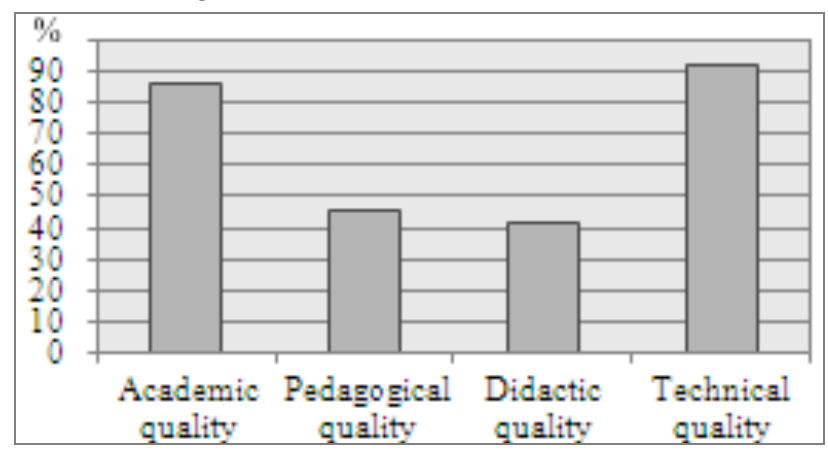

Figure 2. Representative histogram of the evaluated products according to the sections

and technical level, digital educational ressources are insufficient to meet the educational and teaching expectations.

Facing this outcome and so that the educational mission of technology to be accomplished, the production of digital educational resources must take into account the teaching and learning dimension. In addition, the design of such resource requires the mastery of methods, processes and tools specific to digital pedagogy.

\section{CONCLUSIONS AND PROPOSALS}

In this article, we have presented an analysis of the evaluation results of digital educational resources which 
was performed in two stages: an overall evaluation of the quality of the resources and evaluation according to each section (academic, educational, educational and technical). As part of this analysis, we have noticed that a large number of digital educational resources have a low or medium quality.

This result can be interpreted from the fact that, from a pedagogical and didactic perspective, a large number of digital resources do not perform its function (providing space for students to build knowledge, skills and academic knowledge, and developing competencies). Despite their technical and academic quality, these products are merely electronic versions of traditional courses that will not bring anything special to the learner.

In fact, it should be noted that learning with digital resources takes place in a very different context from traditional learning, where human interactions become mediated. While a learner knows implicitly what activities performed with a book or oral discourse, the usual practice does not tell what he/she should do with a digital medium. He does not know what it is possible (annotate? ask questions? synthesize? within what framework? etc.) and he does not know what is expected.

In this new environment where the learner finds $\mathrm{him} /$ herself alone in front of the machine, the need for educational support is of great importance as most digital educational resources are not based on solid teaching and learning foundations.

In this context, to be able to create products that meet most of the digital didactic and pedagogical criteria, explanatory work must be led by the pedagogy agents to guide the manipulation of media by the learner and this work must be done at the design stage. On the one hand, this work must take into account relevant strategies considering all elements of the teaching-learning process. On the other hand, it must evaluate any product before putting it at the hands of learners in order to identify deficiencies and make necessary adjustments during the design process. In this context, several tools (e.g. the designed evaluation grid) and methods [10] can be used for evaluation.

However, teaching and learning models should be established in line with the current practices of educational paradigm that emphasizes the active, reflexive and social learning nature [11]. Learners are not receivers the information provided, but must be seen as active builders of knowledge in the context of a learning community. In this regard, all digital educational resources should reflect the learning process that students develop during different activities: "a situation problem which induces an action and a resolution process in which skills are practiced and new knowledge is identified. This action is followed by activities to consolidate learning and everything is accompanied by a formative assessment "[12].

\section{REFERENCES}

[1] Peraya, D. (1999), "Réalisation d'un dispositif de formation entièrement ou partiellement à distance". Période $1:$ La formation à distance. Un cadre de référence. TECFA.

[2] Ezzahri S., al., (2008), "Elaboration d'un outil d'évaluation de cours de formation continue a distance". ISDM, n³9.

[3] Park I., Hannafin M.-J. (1993), "Empiically-based guidelines for the design of interactive media, Educational Technology Research en Development", Vol.41, Issue 3.

[4] Crozat, S., al., (1999), "EMPI : Une méthode informatisée pour l'évaluation des didacticiels multimédias", RHIM : Revue d'Interaction Homme Machine (ed Europia), Vol 1, n².

[5] Scapin D., Bastien CH. (1997), «Ergonomic criteria for evaluating the ergonomic quality of interactive systems." Behaviour \& Information Technologie, Vol 16, Issue 4-5, pp. 220-231. http://dx.doi.org/10.1080/014492997119806

[6] EL MHOUTI A., NASSEH A., (2013), "Development of a tool for evaluating the quality of digital learning resources," unpublished.

[7] Hû O. (2001), "Contribution à l'évaluation des logiciels multimédias pédagogiques", PhD Thesis, University of Technology of Compiegne, France.

[8] Ecaterina G-P. (2003), "Conception et Evaluation des Environnements pédagogiques sur le Web", Université de Technologie de Compiègne (UTC).

[9] Pierre M., Renata J. (2001), "Outils pour l'analyse de sites Web éducatifs", Module n³, version 4.

[10] Trigano P., Giacomini, E.-P., (April 2004), "CEPIAH a method for Evaluation and Design of Pedagogical Hypermedia," Higher Education in Europe, édition special "Thematic reflexion in Higher Education" vol. XXIX, No.1

[11] Brown A. L., Campione J. C., "Psychological theory and the design of innovative learning environments: on procedures, principles, and systems.” To appear in L. Schauble \& R. Glaser (Eds.), Contributions of Instructional Innovation to Understanding learning. Hillside, NJ: Erlbaum.

[12] Pierre G. al., "Développement d'outils de diagnostic et de remediation immediate au travers d'activités scientifiques au premier degré de l'enseignement secondaire. Présentation de la valise pédagogique Energithèque", Carré des sciences - Institut d'administration scolaire, Université de Mons.

\section{AUTHORS}

EL MHOUTI Abderrahim is with Computer Science Operational Research and Applied Statistics Laboratory, Abdelmalek Essaadi University, Faculty of Sciences, Tetouan, Morocco (e-mail: elmhouti@hotmail.com).

ERRADI Mohamed is with Computer Science Operational Research and Applied Statistics Laboratory, Abdelmalek Essaadi University, Higher Normal School, Tetouan, Morocco (e-mail: erradi_mo@yahoo.fr).

Manuscript received 31 January 2013. Published as resubmitted by the authors 25 April 2013. 